Pesq. Vet. Bras. 37(8):853-858, agosto 2017 DOI: $10.1590 / \mathrm{S} 0100-736 \mathrm{X} 2017000800012$

\title{
Desenvolvimento de caminhar espinal em cães paraplégicos com fraturas e luxações vertebrais toracolombares ${ }^{1}$
}

\author{
Bruno Martins Araújo ${ }^{2 *}$, Thaíza Helena Tavares Fernandes², Durval Baraúna \\ Junior $^{2}$, Marília de Albuquerque Bonelli², Marcela Maria de Almeida Amorim² \\ e Eduardo Alberto Tudury ${ }^{2}$
}

\begin{abstract}
Araújo B.M., Fernandes T.H.T., Baraúna Junior D., Bonelli M.A., Amorim M.M.A. \& Tudury E.A. 2017. [Development of spinal walking in paraplegic dogs with thoracolumbar spinal fractures/luxations.] Desenvolvimento de caminhar espinal em cães paraplégicos com fraturas e luxações vertebrais toracolombares. Pesquisa Veterinária Brasileira 37(8):853-858. Departamento de Medicina Veterinária, Universidade Federal Rural de Pernambuco, Rua Dom Manoel de Medeiros s/n, Dois Irmãos, Recife, PE 52171-900, Brazil. E-mail: bmaraujo85@hotmail.com

Thoracolumbar vertebral fractures and luxations (VFL) are one of the most common neurological disorders in veterinary neurology and one of the most serious and challenging disorders due to the high risk of permanent paralysis, leading many dogs to be euthanized without treatment due to the reports of unfavorable prognosis about ambulation in animals that lost nociception. This study aimed to describe the neurophysiologic bases responsible for the development of the spinal walking and examine in 37 dogs affected with thoracolumbar VFL, data relating to the recovery rate of animals with and without nociceptionIn those without nociception was analyzed the frequency of the spinal walking animals that developed for its appearance, and the average period was established. Regarding the degree of injury to recovery rates, $14 / 37$ dogs $(37.8 \%)$ had nociception, in which the rate of recovery of voluntary ambulation was $100 \%$. While $23 / 37$ dogs $(62.1 \%)$ lost the nociception, where no voluntary ambulation was regained ambulation, occurred death from various causes in seven of these. From 16 dogs without nociception and survivors who underwent conservative or surgical treatment, five (31.25\%) regained the ability to walk without regaining nociception; this was attributed to spinal walking, where the average time for their development was 115 days. According to the results of this study, the single parameter of loss of the nociception should not discourage the therapy, as paraplegic dogs with thoracolumbar VFL can develop involuntary ambulation.

INDEX TERMS: Nervous system, spinal fractures spinal luxations, nociception, spinal walking, dogs.
\end{abstract}

RESUMO.- Fraturas e luxações vertebrais (FLV) toracolombares estão dentre as afecções neurológicas mais frequentes na neurologia veterinária. São um dos distúrbios mais graves e desafiadores, devido ao elevado risco de paralisia permanente, levando muitos animais a serem submetidos à eutanásia, devido ao prognóstico desfavorável nos animais que perderam a nocicepção. Objetivou-se

\footnotetext{
${ }^{1}$ Recebido em 11 de dezembro de 2015.

Aceito para publicação em 2 de dezembro de 2016.

${ }^{2}$ Departamento de Medicina Veterinária, Universidade Federal Rural de Pernambuco, Rua Dom Manoel de Medeiros s/n, Dois Irmãos, Recife, PE 52171-900, Brasil. Pesquisa de mestrado com apoio CNPq. * Autor para correspondência: bmaraujo85@hotmail.com
}

descrever as bases neurofisiológicas responsáveis pelo desenvolvimento do caminhar espinal e analisar, em 37 cães acometidos por FLV toracolombares, os dados referentes à taxa de recuperação dos animais com e sem nocicepção. Naqueles sem nocicepção, analisou-se ainda a frequência dos animais que desenvolveram caminhar espinal e o período médio para seu aparecimento. Em relação ao grau da lesão a as taxas de recuperação, 14/37 animais $(37,8 \%)$ possuíam nocicepção, no qual a taxa de recuperação da deambulação voluntaria e das funções viscerais foi de $100 \%$. Enquanto que $23 / 37$ animais $(62,1 \%)$ perderam a nocicepção, no qual nenhum recuperou a deambulação voluntária, ocorrendo morte por causas diversas em sete destes. Dos 16 animais sem nocicepção sobreviven- 
tes e que foram submetidos ao tratamento conservativo ou cirúrgico, cinco $(31,25 \%)$ readquiriram a capacidade de caminhar (tempo médio de 115 dias) sem recuperar a nocicepção, sendo esta deambulação involuntária atribuída ao caminhar espinal. De acordo com os resultados desta pesquisa, o parâmetro isolado da perda da nocicepção não deve desencorajar a realização da terapia, pois em cães paraplégicos com FLV toracolombares, há possibilidade de ocorrer desenvolvimento de deambulação involuntária.

TERMOS DE INDEXAÇÃO: Sistema nervoso, fraturas e luxações vertebrais, nocicepção, caminhar espinal, cães.

\section{INTRODUÇÃO}

Fraturas e luxações vertebrais (FLV) toracolombares estão dentre as afecções neurológicas mais comumente encontradas na prática clínica e um dos distúrbios neurológicos mais graves, devido ao elevado risco de lesão permanente às estruturas neurais (Braund 1996).

Os pacientes são geralmente acometidos por traumatismos, associados na maioria das vezes a acidentes automobilísticos e exibem hiperpatia e graus variáveis de paresia ou paralisia, dependendo da gravidade da lesão (Weh \& Kraus 2012, Dewey 2014).

Os principais parâmetros para determinar o prognóstico são alguns achados do exame neurológico, sendo o grau de déficit neurológico o parâmetro principal para determinar o prognóstico, que depende principalmente da presença ou ausência de nocicepção nas áreas do corpo caudais à lesão (Braund 1996, Araújo 2013, Araújo et al. 2013).

De acordo com Fletcher (2016), os cães que mantém a nocicepção possuem prognóstico favorável de recuperação funcional e os animais que a perderam em decorrência de FLV apresentam um prognóstico de recuperação desfavorável, pois neste tipo de afecção, a ausência de nocicepção está frequentemente associada com transecção da medula espinal ou desenvolvimento de mielomalácia.

Alguns autores relatam que de forma surpreendente, cães podem recuperar a capacidade de caminhar após secção medular (Shurrager \& Dykman 1951, Freeman 1952, Olby et al. 2003, Lorenz et al. 2011, Gallucci et al. 2015). 0 surgimento de atividade motora nesses cães pode ser indicativo do desenvolvimento de caminhar espinal reflexo, originado do mecanismo de plasticidade neuronal, da formação de circuitos de regulação central medular induzidos por movimentos repetitivos, do desenvolvimento de espasticidade muscular ou da ação de axônios sobreviventes que atravessam o sítio da lesão e permitem que o animal execute movimentos deambulatórios de forma involuntária (Freeman 1952, Dietz 2001, Pearson 2001, Olby et al. 2003, Araújo et al. 2009, Rossignol et al. 2009 \& Martinez et al. 2013, Wittler 2014, Dewey 2016).

Embora diversos mecanismos possam contribuir para o desenvolvimento do caminhar espinal, o de comportamento rítmico dos membros pélvicos é o que melhor explica esse fenômeno (Dietz 2001, Pearson 2001). Este evento corresponde a sequências estereotipadas de movimentos como o caminhar, nos quais a eferência motora é estável, repetível e previsível de um ciclo de atividade para outro (Hill et al. 2012). É estimulado pelo reflexo em massa, uma resposta à estimulação medular excessiva, pela ausência de inibição dos neurônios motores superiores, resultando na ativação de grandes áreas medulares que se caracteriza por movimentação dos membros pélvicos e cauda (Fitzmaurice 2011).

0 reflexo em massa estimula os reflexos de endireitamento, extensor cruzado e de sustentação positiva, que realizados em conjunto e de forma sincronizada pelo estímulo repetitivo, permitem o caminhar espinal. Estudos eletrofisiológicos e histológicos têm demonstrado a criação de circuitos de regulação central medular, que podem ser condicionados por estímulos repetitivos para regular o caminhar espinal (Dietz 2001, Behrman et al. 2006, Barrière et al. 2008, Rossignol et al. 2009, Frigon 2012, Martinez et al. 2013).

0 reflexo em massa elicida, a princípio, o reflexo de endireitamento, que permite com que um animal com secção medular em decúbito realize movimentos involuntários, que o auxiliam a se colocar em estação (Guyton \& Hall 2011). Após posicionamento dos membros pélvicos, estimulações subsequentes em regiões distais destes membros provocam a ocorrência do reflexo extensor cruzado e do reflexo de sustentação positiva. No reflexo extensor cruzado, estímulos sensitivos estimulam a flexão do membro em questão e a extensão do membro contralateral, devido ao mecanismo de inervação recíproca (Guyton 1993, Bouyer \& Rossignol 2003, Rubinson \& Lang 2009, Guyton \& Hall 2011). A resposta do reflexo de extensão positiva é semelhante a do extensor cruzado. No entanto, a extensão do membro oposto ocorre de forma é tão consistente que permite a sustentação de peso. 0 local de pressão, sob um lado do coxim, determina a posição da extensão no membro, num efeito denominado reação magnética, auxiliando na manutenção do equilíbrio (Bouyer \& Rossignol 2003, Guyton \& Hall 2011).

Estímulos sensitivos desempenham um papel importante na estruturação do ciclo do passo, de modo que seja adaptado ao ambiente em constante mudança, uma vez que as conexões com sistema vestibular, cerebelo e córtex cerebral foram interrompidas (Büschges \& El Manira 1998, Bouyer \& Rossignol 2003, Rossignol et al. 2006, Rossignol et al. 2009).

Basicamente dois pontos são utilizados para a caracterização do caminhar espinal: a ausência de nocicepção e o tipo característico de marcha, no qual os membros pélvicos não acompanham os torácicos, com dificuldades bem manifestadas nas curvas e inaptidão de caminhar para trás (Cordeiro 1996).

Objetivou-se descrever as bases neurofisiológicas responsáveis pelo desenvolvimento do caminhar espinal e analisar, em 37 cães acometidos por FLV toracolombares, os dados referentes à taxa de recuperação dos animais com e sem nocicepção, assim como a frequência dos animais que desenvolveram caminhar espinal e o período médio para seu aparecimento, em virtude de relatos na literatura indicando que cães acometidos por fraturas e luxações vertebrais toracolombares, com perda da nocicepção, difi- 
cilmente voltarão a caminhar. Desta forma, tentar diminuir as taxas de eutanásia nesses cães, que chegam a atingir a frequência de $47 \%$ em centros veterinários de referência (Turner 1987, McKee 1990, Selcer et al. 1991, Olby et al. 2003, Bruce et al. 2008, Mendes \& Arias 2012).

\section{MATERIAL E MÉTODOS}

Para o estudo (autorização № 023/2012 da Comissão de Ética no Uso de Animais da Universidade Federal Rural de Pernambuco [UFRPE]), foram analisados os dados de 37 cães, provenientes do atendimento de rotina do Hospital Veterinário da UFRPE, durante o período entre março de 2011 e julho de 2012, sem distinção de sexo, raça ou idade, que apresentaram FLV toracolombares, diagnosticadas por meio de exame neurológico e radiográfico.

Cada paciente passou por uma anamnese detalhada, exame físico e por fim exame neurológico, para se obter o diagnóstico neuroanatômico e a severidade das lesões nervosas. Em seguida, foram solicitados exames complementares de acordo com a necessidade de cada caso. Para avaliação do grau de disfunção neurológica, foram utilizados os parâmetros adaptados de Bruce et al. (2008), sendo os grupos I a IV formados pelos animais que mantiveram a nocicepção intacta e o grupo V, pelos que a perderam.

Inicialmente, os pacientes foram imobilizados em uma tábua acolchoada, a fim de se evitar lesões adicionais à medula espinal. Foram medicados com meloxicam $(0,1 \mathrm{mg} / \mathrm{kg}$, via oral, a cada 24 horas, por sete dias) e cloridrato de tramadol ( $4 \mathrm{mg} / \mathrm{kg}$, via oral, a cada oito horas, por 5 dias), sendo recomendados cuidados gerais de enfermagem e fisioterapia. Em seguida, foram submetidos ao tratamento conservativo ou cirúrgico, de acordo com as necessidades de cada caso.

Foram considerados pertencentes ao grupo de tratamento conservativo aqueles animais que apresentaram déficits neurológicos leves (graus I, II e III), fraturas estáveis e não deslocadas, com ausência ou discreta compressão medular e cães em outros graus que não se apresentaram em condições clínicas de serem submetidos a um procedimento cirúrgico.

Foram considerados pacientes cirúrgicos aqueles com condições orgânicas de serem submetidos ao procedimento cirúrgico e anestésico, que apresentaram déficits neurológicos graves (graus IV e V), lesões instáveis, fraturas deslocadas, evidências de compressão medular e animais que apresentaram piora ante o tratamento conservativo.

Os procedimentos cirúrgicos foram realizados no menor intervalo de tempo possível após o atendimento inicial. A fim de padronizá-los, estes foram realizadas pelos mesmos membros da equipe, efetuando-se descompressão, redução e estabilização vertebral (quando necessário), utilizando-se as técnicas descritas na literatura.

As reavaliações foram realizadas três, sete, 15, 30, 60, 90 e 180 dias após o início do tratamento, para se avaliar a evolução clínica de cada paciente. Durante esse período, foi realizado tratamento fisioterápico conforme indicado por Pearson (2001), Dietz (2001), Behrman et al. (2006) e Gallucci et al. (2015). Iniciou-se com a do tipo passiva, por meio de movimentos de extensão e flexão dos membros pélvicos, imitando o caminhar, durante $15 \mathrm{mi}$ nutos, quatro vezes ao dia. Após estabilização da lesão vertebral, foi recomendada fisioterapia ativa com caminhadas em piso antiderrapante durante 5 minutos, quatro vezes ao dia com apoio de toalha na região ventral do abdome para estimular a movimentação dos membros pélvicos. Toda a fisioterapia foi realizada pelos proprietários dos animais em suas residências. Para monitorar a realização dos exercícios, foi fornecido um diário de registros diários para ser preenchido após a realização das sessões.
Foram considerados animais que desenvolveram caminhar espinal os que apresentaram ausência de nocicepção nos membros pélvicos e cauda, reflexos patelares e flexores dos membros pélvicos normais, reflexo em massa e deambulação com os membros torácicos e pélvicos ao mesmo tempo, sem assistência.

\section{RESULTADOS E DISCUSSÃO}

Os dados referentes ao grau de disfunção neurológica, localização da lesão, tipo de tratamento, o resultado da terapia e ocorrência de caminhar espinal de 37 cães com FLV toracolombares estão expostos no Quadro 1.

Em relação ao grau de disfunção neurológica e as taxas de recuperação, $14 / 37$ animais $(37,8 \%)$ tinham nocicepção intacta, no qual a taxa de recuperação da deambulação voluntária e das funções viscerais foi de $100 \%$. Enquanto que $23 / 37$ animais $(62,1 \%)$ perderam a nocicepção, no qual nenhum recuperou a deambulação voluntária e funções viscerais, ocorrendo óbito por causas diversas em sete destes.

0 prognóstico desfavorável de cães com fraturas vertebrais sem nocicepção pode ser justificado pelo fato da perda da nocicepção estar relacionado à grave lesão da medula espinal (Fletcher 2016), o que pode ser justificado pelos trabalhos de Feeney \& Oliver (1980), McKee (1990) e Selcer et al. (1991), que relataram que os animais que perderam a nocicepção apresentavam no trans-cirúrgico ou na necropsia, secção medular ou sinais de mielomalácia.

Dos 16 animais paraplégicos sem nocicepção sobreviventes e que foram submetidos ao tratamento conservativo ou cirúrgico, cinco $(31,25 \%)$ readquiriram a capacidade de caminhar sem recuperar a nocicepção.

O desenvolvimento de deambulação involuntária nos cães deste trabalho foi atribuído ao caminhar espinal (Shurrager \& Dykman 1951, Olby et al. 2003, Lorenz et al. 2011, Dewey 2016). Segundo Olby et al. (2003), o surgimento de atividade motora em cães que permanecem sem nocicepção está relacionado ao caminhar espinal reflexo. Em seu trabalho que envolvia cães sem nocicepção, vítimas de traumatismo vertebral, a autora menciona que $22 \%$ dos animais não recuperaram a nocicepção, mas readquiriram a capacidade de caminhar. Gallucci et al. (2015) em um estudo com 81 cães sem nocicepção, relatou a ocorrência de desenvolvimento do caminhar espinal em 14 cães $(17,2 \%)$ acometidos por traumatismo vertebral.

No presente estudo, o tempo médio para o desenvolvimento do caminhar espinal foi de 115 dias (Quadro 1), sendo que somente em dois animais a idade era conhecida pelos proprietários, sendo este período de 32 dias no cão de quatro meses e de 120 dias, no cão com dois anos de idade.

Nos animais de idade conhecida, apesar do pequeno número da amostra, o período para o aparecimento do caminhar espinal ocorreu conforme os relatos de Jaggy (2010), ao mencionar que filhotes que sofreram secção medular desenvolveram caminhar espinal em torno de dois meses e que adultos que sofreram o mesmo tipo de trauma necessitaram de vários meses para desenvolvê-lo. Esses dados foram semelhantes os achados de Shurrager \& Dykman (1951), que nos primeiros relatos sobre caminhar espinal em cães, mencionaram que a velocidade desenvolvimento de caminhar espinal ocorreu rapidamente em cães jovens. 
0 reconhecimento do caminhar espinal seguiu os parâmetros de Cordeiro (1996), pois nesses animais a nocicepção estava ausente, os reflexos espinais dos membros pélvicos estavam normais, os membros pélvicos não acompanhavam os torácicos. Houve dificuldades de caminhar nas curvas e inaptidão de caminhar para trás.

Em todos os animais, previamente ao desenvolvimento do caminhar espinal, observou-se o aparecimento do reflexo em massa. Desta forma, este reflexo pode ser considerado um indicativo precoce da possibilidade de desenvolvimento do caminhar espinal em cães. Esta hiperestimulação medular irá ativar e coordenar os reflexos de endireitamento, extensor cruzado, de sustentação positiva, que em conjunto com a impulsão ocasionada pela movimentação voluntaria em sentido cranial dos membros torácicos, possibilitaram o caminhar espinal (Dietz 2001, Pearson 2001, Behrman et al. 2006).

O desenvolvimento do comportamento rítmico gerado pelos reflexos espinais acima citados foi possível pela localização da lesão na região toracolombar da medula espinal, permitindo manter intacto os neurônios motores inferiores da região lombossacral e suas conexões com estímulos sensitivos advindos dos membros pélvicos, permitindo a movimentação involuntária dos membros (Bouyer \& Rossignol 2003, Fitzmaurice 2011, Gallucci et al. 2015).

0 protocolo fisioterapêutico desempenhou um papel importante no desenvolvimento do caminhar espinal nestes cães, corroborando com De Leon (1998), Pearson (2001), Dietz (2001), Behrman et al. (2006), Martinez et al. 2012, Gallucci et al. (2015) e Melo (2015), quando mencionam que o fortalecimento do tônus muscular e o condicionamento dos membros pélvicos por meio de movimentos passivos e repetitivos podem contribuir para a padronização da atividade motora e na formação e estimulação do caminhar espinal. Esses dados confirmam os relatos de Shurrager \& Dykman (1951), que nos primeiros relatos sobre caminhar espinal em cães, já concluíram que o desenvolvimento de caminhar espinal foi diretamente proporcional à quantidade de exercício realizada.

\section{Quadro 1. Dados dos 37 cães acometidos com fraturas e luxações vertebrais com suas idades, grau de disfunção neurológica, localização segmentar da lesão, tipo de tratamento, resultado da terapia e tempo para desenvolvimento do caminhar espinal}

\begin{tabular}{|c|c|c|c|c|c|c|}
\hline Animal & Idade & $\begin{array}{l}\text { Grau de disfunção } \\
\text { neurológica }\end{array}$ & $\begin{array}{l}\text { Localização } \\
\text { da lesão }\end{array}$ & $\begin{array}{l}\text { Tipo de } \\
\text { tratamento }\end{array}$ & $\begin{array}{l}\text { Resultado } \\
\text { da terapia }\end{array}$ & $\begin{array}{l}\text { Tempo de desenvolvimento } \\
\text { do caminhar espinal (Dias) }\end{array}$ \\
\hline 1 & $3 \mathrm{~A}$ & $\mathrm{~V}$ & $\mathrm{~T}_{7}-\mathrm{T}_{8}$ & Conservativo & Não recuperou & - \\
\hline 2 & $\mathrm{NC}$ & $\mathrm{V}$ & $\mathrm{L}_{2}^{7}-\mathrm{L}_{4}^{8}$ & Conservativo & Não recuperou (eutanásia) & - \\
\hline 3 & $9 \mathrm{M}$ & $\mathrm{V}$ & $\mathrm{T}_{7}^{3}-\mathrm{T}_{8}^{4}$ & Conservativo & Não recuperou (eutanásia) & - \\
\hline 4 & NC & IV & $\mathrm{T}_{12}^{7}-\mathrm{T}_{13}^{8}$ & Cirúrgico & Recuperação total & - \\
\hline 5 & NC & III & $\mathrm{T}_{12}^{12}-\mathrm{T}_{13}^{13}$ & Conservativo & Recuperação total & - \\
\hline 6 & $8 \mathrm{~A}$ & II & $\mathrm{T}_{1}^{12}-\mathrm{T}_{2}^{13}$ & Conservativo & Recuperação total & - \\
\hline 7 & $3 \mathrm{~A}$ & II & $\mathrm{L}_{3}^{1}-\mathrm{L}_{4}^{2}$ & Conservativo & Recuperação total & - \\
\hline 8 & NC & III & $\mathrm{T}_{11}^{3}-\mathrm{T}_{12}^{4}$ & Conservativo & Recuperação total & - \\
\hline 9 & $8 \mathrm{~A}$ & $\mathrm{~V}$ & $\mathrm{~T}_{11}^{11}-\mathrm{T}_{12}^{12}$ & Conservativo & Não recuperou (eutanásia) & - \\
\hline 10 & $6 \mathrm{~A}$ & $\mathrm{~V}$ & $\mathrm{~T}_{12}^{11}-\mathrm{T}_{13}^{12}$ & Conservativo & Não recuperou & - \\
\hline 11 & $5 \mathrm{M}$ & IV & $\mathrm{T}_{12}^{12}-\mathrm{T}_{13}^{13}$ & Cirúrgico & Recuperação parcial (óbito por IRA) & А) \\
\hline 12 & $10 \mathrm{~A}$ & III & $\mathrm{T}_{9}-\mathrm{T}_{10}^{13}$ & Conservativo & Recuperação total & - \\
\hline 13 & $\mathrm{NC}$ & V & $\mathrm{T}_{12}-\mathrm{T}_{13}$ & Conservativo & Não recuperou (eutanásia) & - \\
\hline $14^{*}$ & $2 \mathrm{~A}$ & $\mathrm{~V}$ & $\mathrm{~T}_{12}^{12}-\mathrm{T}_{13}^{13}$ & Cirúrgico & Não recuperou & 120 dias \\
\hline 15 & $8 \mathrm{~A}$ & III & $\mathrm{T}_{12}^{12}-\mathrm{T}_{13}^{13}$ & Conservativo & Recuperação total & - \\
\hline 16 & $20 \mathrm{~A}$ & $\mathrm{~V}$ & $\mathrm{~T}_{12}^{12}-\mathrm{T}_{13}^{13}$ & - & Eutanásia sem tratamento & - \\
\hline 17 & $1 \mathrm{~A}$ & $\mathrm{~V}$ & $\mathrm{~L}_{3}-\mathrm{L}_{4}$ & Conservativo & Não recuperou & - \\
\hline 18 & $2 \mathrm{~A}$ & V & $\mathrm{T}_{13}-\mathrm{l}_{1}$ & - & Óbito (ruptura de vesícula urinária) & a) \\
\hline 19 & $15 \mathrm{~A}$ & $\mathrm{~V}$ & $\mathrm{~T}_{11}^{13}-\mathrm{T}_{12}^{1}$ & Cirúrgico & Não recuperou & - \\
\hline 20 & $3 \mathrm{~A}$ & IV & $\mathrm{T}_{11}^{11}-\mathrm{T}_{12}^{12}$ & Cirúrgico & Recuperação total & - \\
\hline 21 & $\mathrm{NC}$ & III & $\mathrm{L}_{2}-\mathrm{L}_{3}$ & Cirúrgico & Recuperação total & - \\
\hline 22 & $12 \mathrm{~A}$ & $\mathrm{~V}$ & $\mathrm{~T}_{12}-\mathrm{T}_{13}$ & - & Óbito (trauma torácico) & - \\
\hline 23 & $\mathrm{NC}$ & V & $\mathrm{T}_{13}-\mathrm{L}_{1}$ & Cirúrgico & Não recuperou & - \\
\hline 24 & $\mathrm{NC}$ & III & $\mathrm{T}_{11}^{13}-\mathrm{T}_{12}^{1}$ & Conservativo & Recuperação total & - \\
\hline $25^{*}$ & $\mathrm{NC}$ & $\mathrm{V}$ & $\mathrm{T}_{12}^{11}-\mathrm{T}_{13}^{12}$ & Cirúrgico & Não recuperou & 120 dias \\
\hline $26^{*}$ & NC & V & $\mathrm{T}_{11}^{12}-\mathrm{T}_{12}^{13}$ & Conservativo & Não recuperou & 130 dias \\
\hline 27 & $3 \mathrm{~A}$ & V & $\mathrm{T}_{11}^{11}-\mathrm{T}_{12}^{12}$ & Cirúrgico & Não recuperou & - \\
\hline 28 & $1 \mathrm{~A}$ & $\mathrm{~V}$ & $\mathrm{~T}_{9}-\mathrm{T}_{10}^{12}$ & Conservativo & Não recuperou & - \\
\hline 29 & $\mathrm{NC}$ & IV & $\mathrm{L}_{1}-\mathrm{L}_{2}$ & Cirúrgico & Recuperação total & - \\
\hline 30 & NC & V & $\mathrm{T}_{13}^{1}-\mathrm{L}_{1}^{2}$ & Conservativo & Não recuperou & - \\
\hline $31^{*}$ & $4 \mathrm{M}$ & V & $\mathrm{T}_{12}^{13}-\mathrm{T}_{13}^{1}$ & Cirúrgico & Não recuperou & 32 dias \\
\hline 32 & $8 \mathrm{M}$ & V & $\mathrm{L}_{1}-\mathrm{L}_{2}$ & Conservativo & Não recuperou & - \\
\hline 33 & $8 \mathrm{M}$ & II & $\mathrm{L}_{3}-\mathrm{L}_{4}$ & Conservativo & Recuperação total & - \\
\hline 34 & $8 \mathrm{~A}$ & I & $\mathrm{T}_{12}-\mathrm{T}_{13}$ & Conservativo & Recuperação total & - \\
\hline $35^{*}$ & NC & V & $\mathrm{T}_{11}^{12}-\mathrm{T}_{12}^{13}$ & Cirúrgico & Não recuperou & 180 dias \\
\hline 36 & $8 \mathrm{~A}$ & $\mathrm{~V}$ & $\mathrm{~T}_{1}-\mathrm{T}_{12}$ & Cirúrgico & Não recuperou & - \\
\hline 37 & $\mathrm{NC}$ & V & $\mathrm{T}_{13}-\mathrm{L}_{1}$ & Cirúrgico & Não recuperou & - \\
\hline
\end{tabular}

A: Anos; M: meses; IRA: Insuficiência renal aguda.; NC: Não conhecida

*Animais que desenvolveram caminhar espinal. 
A taxa total de eutanásia no presente estudo foi de 13,5\% (5 animais), resultado inferior aos de outros autores descritos na literatura (Turner 1987, McKee 1990, Selcer et al. 1991, Olby et al. 2003, Bruce et al. 2008, Mendes \& Arias 2012), permitindo com que a maioria dos animais sem nocicepção que não recuperaram a deambulação voluntária permanecessem vivos e tivessem continuidade do seu tratamento de reabilitação. Desta forma, a frequência inferior de desenvolvimento de caminhar espinal descrito em outros trabalhos pode ser devido à alta taxa de eutanásia que foi praticada nos cães com FLV sem nocicepção.

0 protocolo de tratamento do presente estudo permitiu uma porcentagem alta de desenvolvimento de caminhar espinal (31,5\%), em relação a outros estudos semelhantes (Olby et al. 2003, Mendes \& Arias 2012, Gallucci et al. 2015), assinalando que esta abordagem terapêutica deve ser rotineira nos cães com FLV que perderam a nocicepção.

Todos os animais paraplégicos que desenvolveram caminhar espinal necessitaram de cuidados de enfermagem intensivos permanentes para manutenção das funções viscerais. Esses cuidados consistiram em esvaziamento vesical e intestinal, por meio de massagem abdominal e fornecimento de dieta adequada; prevenção de assaduras na pele e ulcerações cutâneas por decúbito, por meio de utilização de fraldas descartáveis, limpeza cutânea constante de urina e fezes, mudança de decúbito a cada quatro horas e a realização de fisioterapia passiva e ativa, para evitar atrofia, contratura muscular e perda da função articular e neuromuscular. Após o desenvolvimento do caminhar espinal, mesmo com a necessidade de cuidados gerais de enfermagem e conhecimento da ausência de recuperação da deambulação voluntária, todos os proprietários ficaram satisfeitos com o resultado final.

\section{CONCLUSÃO}

O parâmetro isolado da perda da nocicepção, em cães paraplégicos por fraturas e luxações vertebrais toracolombares, não deve desencorajar a realização da terapia conservativa ou cirúrgica indicada para cada caso, pois conforme os resultados deste trabalho, mesmo que não ocorra a recuperação da deambulação voluntária, há possibilidade de desenvolvimento do caminhar espinal.

\section{REFERÊNCIAS}

Araújo B.M., Arias M.V.B \& Tudury E.A. 2009. Paraplegia aguda com perda da percepção de dor profunda em cães: revisão de literatura. Clínica Vet. 14(81):70-82.

Araújo B.M. 2013. Fraturas e Luxações Vertebrais Toracolombares em Cães: observações clínico-cirúrgicas. Dissertação de Mestrado em Ciência Veterinária, Departamento de Medicina Veterinária, Universidade Federal Rural de Pernambuco, Recife. 103p.

Araújo B.M., Fernandes T.H.T., Souza A.F.A., Diogo C.C., Santos C.R.O., Amorim M.M.A. \& Tudury E.A. 2013. Determinação do prognóstico em 37 cães com fraturas e luxações vertebrais toracolombares por meio da presença da postura de Schiff-Sherrington, reflexo extensor cruzado e o sinal de Babinski. Anais XIII Jornada de Ensino, Pesquisa e Extensão (Jepex), 2012, Recife. (Resumo expandido)

Barrière G., Leblond H., Provencher J. \& Rossignol S. 2008. Prominent role of the spinal central pattern generator in the recovery of locomotion after partial spinal cord injuries. J. Neurosci. 28(15):3976-3987.
Behrman A.L., Bowden M.G. \& Nair P.M. 2006. Neuroplasticity after spinal cord injury and training: walking recovery an emerging paradigm shift in rehabilitation and walking recovery. Phys. Ther. 86(14):1406-1425.

Bouyer L.J.G. \& Rossignol S. 2003. Contribution of cutaneous inputs from the hindpaw to the control of locomotion. 2. Spinal cats. J. Neurophysiol. 90:3640-3653.

Braund K.J. 1996. Traumatismo agudo da medula espinhal, p.1311-1325. In: Bojrab M.J. (Eds), Mecanismos da Moléstia na Cirurgia de Pequenos Animais. Manole, São Paulo.

Bruce C.W., Brisson B.A. \& Gyselinck K. 2008. Spinal fractures in dogs and cats: a retrospective evaluation of 95 cases. Vet. Comp. Orthop. Traumatol. 21(3):280-284.

Büschges A. \& El Manira A. 1998. Sensory pathways and their modulation in the control of locomotion. Curr. Opin. Neurobiol. 8(6):733-739.

Cordeiro J.M.C.O. 1996. Exame Neurológico de Pequenos Animais. EDUCAT, Pelotas. 270p.

De Leon R.D., Hodgson J.A., Roy R.R. \& Edgerton V.R. 1998. Locomotor capacity attributable to step training versus spontaneous recovery after spinalization in adult cats. J. Neurophysiol. 79:1329-1340.

Dewey C.W. 2014. Cirurgia da coluna toracolombar, p.1508-1528. In: Fossum T.W. (Ed.), Cirurgia de Pequenos Animais. 4⿳亠丷a ed. Elsevier, Rio de Janeiro.

Dewey C.W. 2016. Lesion localization: functional and dysfunctional neuroanatomy, p.29-52. In: Dewey C.W \& Da Costa R.C. (Eds), Practical Guide to Canine and Feline Neurology. $3^{\text {rd }}$ ed. Wiley-Blackwell, Iowa.

Dietz V. 2001. Spinal cord lesion: effects of and perspectives for treatment. Neural Plast. 8(1):83-90.

Feeney D.A. \& Oliver J.E. 1980. Blunt spinal trauma in the dog and cat: neurologic, radiologic, and therapeutic correlations. J. Am. Vet. Med. Assoc. 16(664):664-668.

Fitzmaurice S. 2001. Neurologia em pequenos animais. Elsevier, Rio de Janeiro. 332p.

Fletcher D.J., Dewey C.W \& Da Costa R.C. 2016. Spinal trauma management, p.423-436. In: Dewey C.W. \& Da Costa R.C. (Eds), Practical Guide to Canine and Feline Neurology. 3rd ed. Wiley-Blackwell, Iowa.

Freeman L.W. 1952. Return of function after complete transection of the spinal cord of the rat, cat and dog. Ann. Surg. 136(2):193-205.

Frigon A. 2012. Central pattern generators of the mammalian spinal cord. Neuroscientist 18(1):56-69.

Gallucci A., Gandini G., Menchetti M., Gagliardo T., Pietra M., Cardinali M. \& Dragone L. 2015. Acquisition of involuntary spinal locomotion (Spinal Walking) in dogs with irreversible thoraco-lumbar spinal cord lesion: a retrospective study on 81 dogs. Proc. 28th European Veterinary Congress on Movement Disorders ESVN-ECVN Congress, Amsterdam, p.52. (Abstract)

Guyton A.C. 1993. Funções motoras da medula espinhal; os reflexos medulares, p.177-187. In: Guyton A.C. (Ed.), Neurociência Básica: anatomia e fisiologia. $2^{\mathrm{a}}$ ed. Guanabara Koogan, Rio de Janeiro.

Guyton A.C. \& Hall J.E. 2011. Funções motoras da medula espinhal; os reflexos espinhais, p.693-704 . In: Hall J.E. (Ed.), Tratado de Fisiologia Médica. $12^{\mathrm{a}}$ ed. Elsevier, Rio de Janeiro.

Hill R.W., Wyse G.A. \& Anderson M. 2012. Fisiologia Animal. $2^{\text {a }}$ ed. Artmed, Porto Alegre. 894p.

Jaggy A. 2010. Small Animal Neurology: an illustrated text. Schlütersche, Hannover. 570p.

Lorenz M.D., Coates J.R. \& Kent M. 2011. Handbook of Veterinary Neurology. 5 th ed. Elsevier, St Louis. 545 p.

Martinez M., Delivet-Mongrain H., Leblond H. \& Rossignol S. 2012. Effect of locomotor training in completely spinalized cats previously submitted to a spinal hemisection. J. Neurosci. 32(32):10961-10970

Martinez M., Delivet-Mongrain H. \& Rossignol S. 2013. Treadmill training promotes spinal changes leading to locomotor recovery after partial spinal cord injury in cats. J. Neurophysiol. 109(2):2909-2922.

McKee W.M. 1990. Spinal trauma in dogs and cats: a review of 51 cases. Vet. Rec. 126(6):285-289. 
Melo M.R.M. 2015.Medicina física e reabilitação de paraplegias de grau 0 em animais de companhia. Dissertação de Mestrado em Medicina Veterinária, Faculdade de Medicina Veterinária, Universidade Lusófona de Humanidades e Tecnologias, Lisboa. 107p.

Mendes D.S. \& Arias M.V.B. 2012. Traumatismo da medula espinhal em cães e gatos: estudo prospectivo de 57 casos. Pesq. Vet. Bras. 32(12):13041312.

Olby N., Levine J., Harris T., Muñana K., Skeen T. \& Sharp N. 2003. Long-term functional outcome of dogs with severe injuries of the thoracolumbar spinal cord: 87 cases (1996-2001). J. Am. Vet. Med. Assoc. 222(6):762-769.

Pearson K.G. 2001. Could enhanced reflex function contribute to improving locomotion after spinal cord repair?. J. Physiol. 553(1):75-81.

Rossignol S., Barrière G., Alluin O. \& Frigon A. 2009. Re-expression of locomotor function after partial spinal cord injury. Physiology 24:127-139.

Rossignol S., Dubuc R. \& Gossard J.P. 2006. Dynamic sensorimotor interactions in locomotion. Physiol. Rev. 86:89-154.
Rubinson K. \& Lang E.J. 2009. Organização da função motora, p.157-200. In: Koeppen B.M. \& Stanton B.A. (Eds), Fisiologia. 6 ${ }^{\underline{a}}$ ed. Elsevier, Rio de Janeiro.

Selcer R.R., Bubb W.J. \& Walker T.L. 1991. Management of vertebral column fractures in dogs and cats: 211 cases (1977-1985). J. Am. Vet. Med. Assoc. 198(11):1965-1968.

Shurrager P.S. \& Dykman R.A. 1951. Walking spinal carnivores. J. Comp. Physiol. Psych. 44(3):252-262.

Turner W.D. 1987. Fractures and fractures-luxations of the lumbar spine: a retrospective study in the dog. J. Am. Vet. Med. Assoc. 23(459):459-464.

Weh M. \& Kraus K.H. 2012. Spinal fractures and luxations, p.487-503. In: Tobias K.M. \& Johnston S.A. (Eds), Veterinary Surgery: small animals. Saunders, Elsevier.

Wittler L.F.F. 2014. Neuroprosthetic rehabilitation and translational mechanism after severe spinal cord injury. Thèse du Programme Doctoral en Neurosciences, École Polytechnique Fédérale de Lausanne, Lausanne, Suisse. 242p. 